\title{
Human membrane protein Tim-3 facilitates hepatitis A virus entry into target cells
}

\author{
LILI SUI $^{1 *}$, NAN LI ${ }^{1}$, WENYUAN ZHANG ${ }^{2}$, YONG CHEN ${ }^{2}$, YUANYUAN ZHENG $^{1}$, TAO WAN $^{1}$, \\ WEIPING ZHANG ${ }^{1}$, YADONG YANG ${ }^{2}$, GUOJIAN FANG ${ }^{2}$, JIANGSEN MAO ${ }^{2}$ and XUETAO CAO ${ }^{1}$ \\ ${ }^{1}$ Institute of Immunology, Second Military Medical University, Shanghai 200433; \\ ${ }^{2}$ Department of Biological Engineering, Zhejiang Academy of Medical Science, Hangzhou 310013, P.R. China
}

Received January 9, 2006; Accepted February 27, 2006

\begin{abstract}
In this study, a cellular surface membrane protein of immunoglobulin (Ig) superfamily (IgSF) was identified from a human dendritic cell (DC) cDNA library by largescale random sequencing, which is identical to previously reported Tim-3 (T-cell Ig- and mucin-domain-containing molecule 3 ). Recent data have suggested the association of the 281-residue mouse Tim-3 molecule with Th1-related T cell responses and disease in mice. Human Tim-3 is a 301residue type I membrane protein whose extracellular region contains a Cys-rich Ig-like domain and a mucin domain, the characteristics of Tim proteins. It shows significant homology to human hepatitis A virus (HAV) cellular receptor-1 (HuHAVcr-1)/Tim-1. Human Tim-3 mRNA was highly expressed in monocytes or monocyte-derived cells, and the expression level decreased when DC underwent maturation and activation. There is no previous report on the biological functions of human Tim-3, especially the involvement in virus infection. We demonstrated that HeLa cells, which are refractory to HAV infection, acquired a limited susceptibility to HAV infection after stably overexpressing human Tim-3 as confirmed by Western blot analysis using anti-Tim-3 antibody, but Tim-3-Fc fusion protein had no direct HAV-binding activity. The results indicated that human Tim-3 can promote HAV entry into target cells but itself may not function as a cellular receptor of HAV.
\end{abstract}

\section{Introduction}

HAV (hepatitis A virus), the causative agent of hepatitis in human, produces substantial morbidity and mortality, with an estimated 125,000-200,000 infections occurring each year (1). $\mathrm{HAV}$ is an atypical member of the Picornaviridae, and is the

Correspondence to: Dr Xuetao Cao, Institute of Immunology, Second Military Medical University, 800 Xiangyin Road, Shanghai 200433, P.R. China

E-mail: caoxt@public3.sta.net.cn

${ }^{*}$ Contributed equally

Key words: dendritic cells, membrane protein, hepatitis A virus only member of the hepatovirus genus of the Picornaviridae, a family of small, nonenveloped, positive-strand RNA viruses that include human pathogens such as poliovirus (PV) and rhinovirus as well as animal pathogen such as foot-and-mouth disease virus and encephalomyocarditis virus (2). Most wildtype strains of HAV do not grow in cell culture; however, attenuated variants that grow efficiently in primate cell culture have been isolated on serial passaging of the virus (3-5). HAV has also been adapted to grow in guinea pig, pig, and dolphin cell culture, indicating that the cellular factors required for HAV replication are not restricted to primates $(6,7)$. Like other picornaviruses, the first step in the life cycle of HAV is its interaction with a cellular receptor that allows it to enter the cells. Using the protective monoclonal antibody 190/4 as a probe, Kaplan et al identified the HAV cellular receptor-1 (HAVcr-1) in African green monkey cells as a receptor of HAV $(8,9)$. Later, the same research group proved the existence of human homologue of HAVcr-1. It was named HuHAVcr-1, which is the first identified attachment and cellular receptor of HAV in humans. HuHAVcr-1 encodes a polypeptide of 359 amino acids, with typical features of type I integral-membrane glycoprotein (10). The extracellular domain of HuHAVcr-1 contains an N-terminal cysteine-rich region, which shares homology to members of the immunoglobulin superfamily (IgSF), followed by a threonine-, serine-, and proline-rich (TSP-rich) region, which is characteristic of $\mathrm{O}$-glycosylated mucin-like glycoproteins. The N-terminal cysteine-rich region is sufficient for binding and neutralization of HAV (11).

HuHAVcr-1 is also called human Tim-1, which belongs to the T-cell immunoglobulin- and mucin-domain containing molecule (Tim) protein family. Tim proteins are mainly expressed on $\mathrm{T}$ cells and characterized by a conserved set of immunoglobulin and mucin domains. McIntire et al originally identified a Tim locus conferring susceptibility to mouse allergen-induced airway hypersensitivity (12) and found remarkable numbers of sequence polymorphisms within human Tim-1/HuHAVcr-1. The human homologue of Tim-2 has not yet been identified. Mouse Tim-3 was identified in a screen for Th1 cell-reactive monoclonal antibodies as a Th1 cell-specific surface protein (13). It has been shown that mouse Tim-3 is involved in the interactions between Th1 cells and macrophages, which results in the expansion and activation of macrophages. As a homologue of mouse Tim-3, 
the sequence of human Tim-3 was also reported without further functional characterization (13). However, none of these Tim proteins have been reported to be related to HAV infection except Tim-1/HuHAVcr-1.

By large-scale random sequencing of human dendritic cell (DC) cDNA library, we have identified several novel human genes with biological functions (14-16). One of the full-length cDNAs encodes a 301-residue type I integral membrane glycoprotein. The extracellular domain of the novel molecule consists of an $\mathrm{N}$-terminal cysteine-rich region with homolog to members of the immunoglobulin (Ig) superfamily (IgSF). Blast search revealed that the membrane molecule is identical to the previously reported human Tim-3, and also shares 57\% homology with HuHAVcr-1/Tim-1. Therefore, we speculated that this membrane molecule might be another receptor for HAV. While our experiments showed that this membrane molecule did not bind to HAV, suggesting it was not a receptor for $\mathrm{HAV}$, it was able to promote HAV entry into target cells. These data indicate that human Tim-3 might act as a cofactor to promote HAV entry into target cells, but itself may not function as a cellular receptor of HAV.

\section{Materials and methods}

Cell lines and culture. The cell lines were obtained from ATCC. All cell lines were maintained in RPMI-1640 (Hyclone) or Dulbecco's modified Eagle's medium (Hyclone) supplemented with $10 \%$ fetal calf serum (Hyclone) as recommended. Human dendritic cells (DCs) were generated from human peripheral monocytes by culturing with GM-CSF (50 ng/ml) and IL-4 (10 ng/ml) (Sigma) as described previously $(15,16)$. After 6 days of culture, DC were harvested for subsequent experiments. For detection of inducible expression pattern of human Tim-3, human immature monocyte-derived DC were treated with LPS ( $1 \mu \mathrm{g} / \mathrm{ml}$, Sigma), CpG-containing oligonucleotides (30 $\mu \mathrm{g} / \mathrm{ml})$, IFN-ß (100 U/ml, R\&D) or TNF $\alpha$ (10 ng/ml, Peprotech) for $12 \mathrm{~h}$.

Large-scale sequencing of a human DC cDNA library and isolation of full-length human Tim-3 cDNA. By large-scale sequencing of a human dendritic cell cDNA library as described previously $(15,16)$, the full-length of cDNA clone HTD2B5 was isolated to potentially encode an immunoglobulin (Ig) superfamily (IgSF) protein with an Ig-like domain, sharing homology with huHAVcr-1 and may function as novel membrane receptor, which can facilitate HAV entry into target cells. The sequence of human Tim-3 was deposited in GenBank on April 3, 2000, with acc. no.: AF251707. During our investigation, the sequence of human Tim-3, an identical molecule was reported (13) with no functional description, which was deposited in GenBank on February 11, 2002.

Northern blot analysis. Northern blot filter containing human poly(A) ${ }^{+}$RNA ( $2 \mu \mathrm{g} /$ lane) from various tissues was purchased from BD Clontech. The SalI/NotI fragment of human Tim-3 cDNA was used as templates for probe synthesis. The filters were hybridized with the ${ }^{32} \mathrm{P}-$ labeled human Tim-3 cDNA probe in ExpressHyb hybridization solution (BD Clontech) according to the manufacturer's instructions. The filter was re-probed with a human $\beta$-actin cDNA probe.

RT-PCR analysis. Total cellular RNA extraction and first strand cDNA synthesis was performed as described previously (16). RT-PCR with human Tim-3 primer 5' AGCTCCATGT TTTCACATCTTCC (sense) and 5' TGGTTGGATCTATG GCATTGC (antisense) were performed using Exo Taq polymerase (Takara). Synthesis of cDNA was checked by B-actin amplification. The amplification products were resolved on $2 \%$ agarose gel.

Expression of GST fusion protein and generation of polyclonal antibody against human Tim-3. The cDNA encoding the N-terminal first 180 residues of human Tim- 3 was cloned into the prokaryotic expression vector pGEX-2T (Pharmacia, Piscataway, NJ) for GST fusion protein expression. The soluble GST-Tim-3 fusion protein expressed in E. coli BL21 was affinity-purified with glutathione Sepharose 4B (Pierce, Rockford, IL). Polyclonal antibody to recombinant human Tim-3 (anti-Tim-3) was raised in rabbits immunized with the purified GST-Tim-3 fusion protein.

Plasmid construction and cell transfection. To generate a human Tim-3 mammalian expression vector, the code region of human Tim-3 was inserted into pcDNA3.1/myc-His (-)B vector (Invitrogen). The human Tim-3 expression vector and the mock vector pcDNA3.1/myc-His (-)B were transfected into $\mathrm{HeLa}$ cells using Lipofectamine reagent (Invitrogen) according to the manufacturer's instructions. After 2 weeks of screening in the presence of $800 \mu \mathrm{g} / \mathrm{ml} \mathrm{G} 418$ (Calbiochem, La Jolla, CA), stably transfected clone designated HeLa-Tim-3 and HeLa-mock was obtained, and the expression of human Tim-3 protein was confirmed by Western blot analysis.

Western blot analysis. HeLa-Tim-3 and HeLa-mock transfectants were harvested and lyzed with cell lysis buffer (Cell Signaling) containing PMSF $(100 \mu \mathrm{g} / \mathrm{ml})$ and aprotinin $(1 \mu \mathrm{g} /$ $\mathrm{ml})$. An equal amount of whole lysates were separated on SDS-PAGE and transferred onto a nitrocellulose membrane (Amersham). For detection of human Tim-3 protein, blots were probed for $1 \mathrm{~h}$ with anti-Tim-3 polyclonal antibody and then incubated with horseradish peroxidase (HRP)conjugated anti-rabbit IgG (Santa Cruz). The proteins were visualized using Chemiluminescence luminol reagent (Santa Cruz).

Preparation of HAV. Most wild-type strains of HAV do not grow in cell culture; however, attenuated variants that grow efficiently in primate cell culture have been isolated on serial passaging of the virus. Strain $\mathrm{H} 2(17,18)$, an attenuated live hepatitis A virus was derived from the fecal specimen of a patient with hepatitis, after isolation, to grow in human lung diploid cells (KMB17) at a low temperature. Viral stock was prepared as follows. Fresh KMB17 cells were infected with a multiplicity of infection of HAV or not, incubated for 28 days at $35^{\circ} \mathrm{C}, 5 \% \mathrm{CO}_{2}$ incubator. Cells were scrapped with phosphate-buffered saline (PBS), pelleted, and re-suspended in cell lysis buffer (10 mM NaCl, $10 \mathrm{mM}$ Tris-Cl, $\mathrm{pH} 7.4,1 \%$ NP 40). Supernatants containing cytoplasmic extracts were 


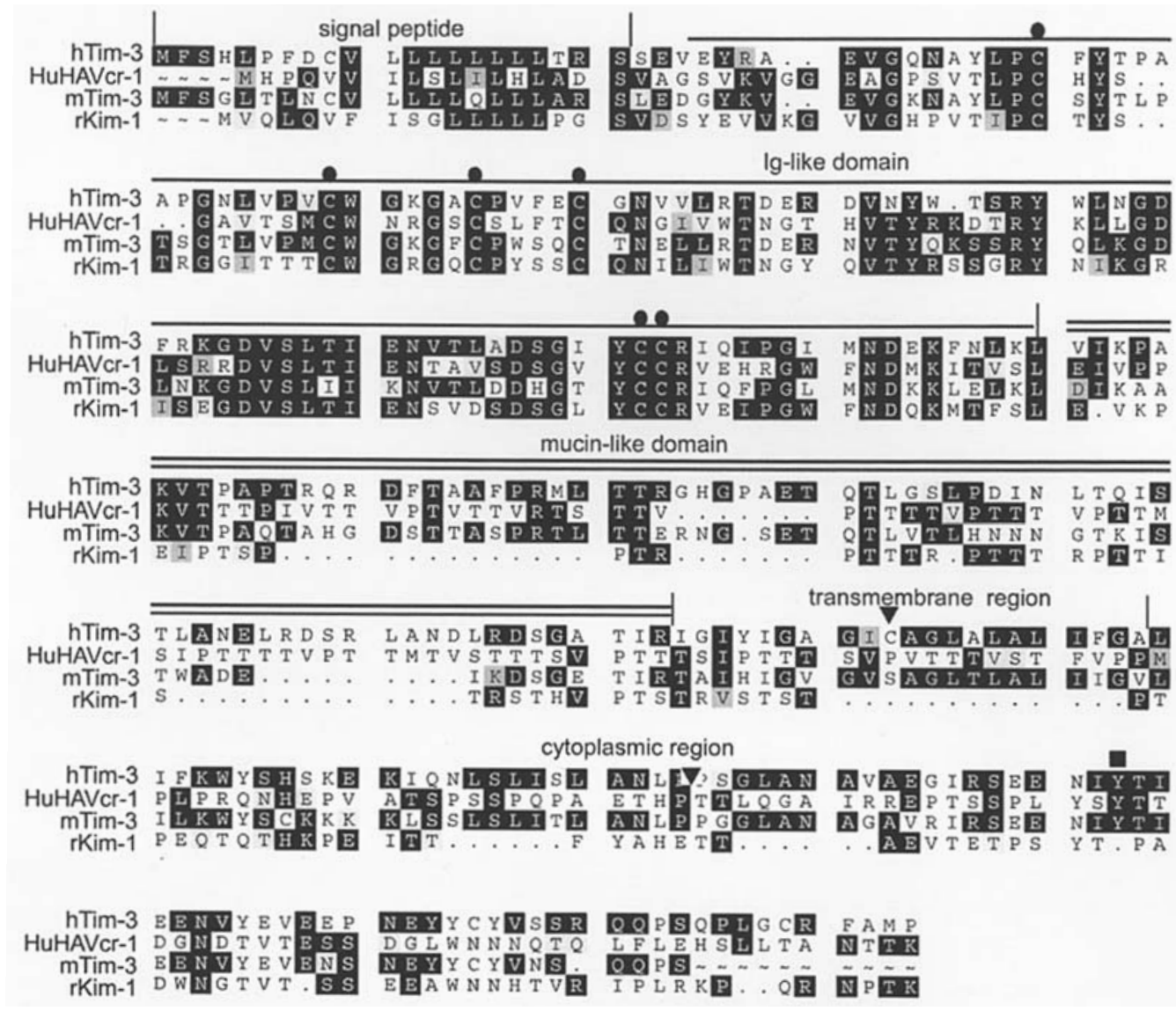

Figure 1. Multiple alignment of human Tim-3 with closely related Tim family members. Alignment was performed with the GCG package and minimally adjusted manually. Identical residues are boxed in black, and similar residues are in gray. Approximate domain boundaries are indicated for signal peptide, Ig-like domain, mucin domain, transmembrane region and cytoplasmic region. The six cysteines of the Cys-rich region are marked with solid circles. The arrowhead indicates the conserved Cys of the transmembrane region. A putative tyrosine phosphorylation is shown by a solid box. The accession no. of human Tim-3, human HuHAVcr-1, mouse HAVcr-2/Tim-3 (mTim-3) and rat Kim-1 (rKim-1) are: AF251707, AAC39862, AAL65156 and AAH61820, respectively.

obtained by centrifugation at $12000 \mathrm{~g}, 5 \mathrm{~min}$ and used as viral stock after titration of $>10^{8}$. Non-infected cell lysis supernatants were used as mock infectant in HAV infectivity assay.

HAV infectivity assay. HeLa and KMB17 cells (as positive control) grown in six-well culture plates to $80 \%$ confluency were infected with $10^{7}-10^{8}$ TCID of $\mathrm{HAV} \mathrm{H}_{2}$ strain or mock infectant for $6 \mathrm{~h}$ at $35^{\circ} \mathrm{C}$ under $5 \% \mathrm{CO}_{2}$. After being washed 3 times, cells were incubated for 3 or 7 days at $35^{\circ} \mathrm{C}$ under $5 \%$ $\mathrm{CO}_{2}$. HAV within the infected cells was released by 3 freezethaw cycles, cell debris was separated by centrifugation at $12000 \mathrm{~g}, 2 \mathrm{~min}$, and the supernatants were collected for the next analysis. For HAV detection by ELISA, PVC plates were coated with goat anti-human $\operatorname{IgM} \mu$-chain-specific Ab and incubated with human anti-HAV serum. Then the collected cell lysis supernatants was added and incubated for 18-22 h, washed extensively with PBS, and treated with human HRP-labeled anti-HAV Ab for $2 \mathrm{~h}$. The reaction was visualized with tetramethyl-benzidine (TMB) substrate and absorbance at $595 \mathrm{~nm}$ was determined in an ELISA reader (Bio-Rad).

Expression of soluble eTim-3-Fc fusion protein. Coding regions of the extracellular domain of human Tim-3 and human IgG1 $\mathrm{Fc}$ fragment were cloned in frame into pcDNA3.1/mic-His (-)B for expression of the secreted human extracellular Tim-3-Fc fusion protein (eTim-3-Fc). COS7 cells were transfected with Lipofectamine, the culture supernatant was harvested $48 \mathrm{~h}$ post-transfection, and the soluble eTim-3-Fc fusion protein was purified using Affi-Gel protein A-agarose columns (BioRad, Hercules, CA).

HAV-binding assay of eTim-3-Fc fusion protein. For binding assays, different amounts of purified eTim-3-Fc, CTLA4-Fc, human IgG or human HAV IgM were bound to protein ATrisacryl beads (Pierce) in PBS containing 2\% ovalbumin (Sigma). Purified HAV viral stock was added and the mixture was incubated with rotation overnight at $4^{\circ} \mathrm{C}$. After 3 PBS washings at $4^{\circ} \mathrm{C}, \mathrm{HAV}$-bound beads were identified by Western blot analysis using anti-HAV IgG polyclonal antibody.

\section{Results}

Identification and sequence analysis of human Tim-3. An EST for a homologue of hepatitis A virus (HAV) receptor was originally isolated from a human DC cDNA library by largescale random sequencing. The 2320-bp full-length cDNA of the novel protein encoded a single 301 amino acid open reading frame with a theoretic molecular mass of 33394 Dalton and an isoelectric point of 5.54. A BLAST search of NCBI protein 


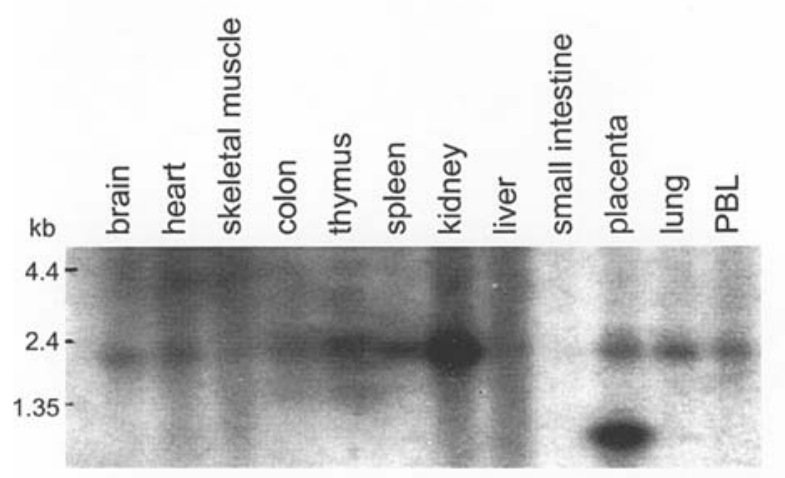

B

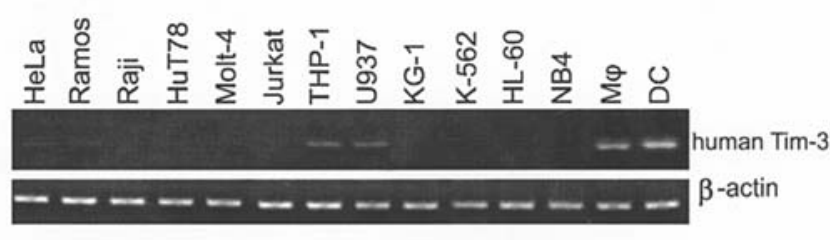

C

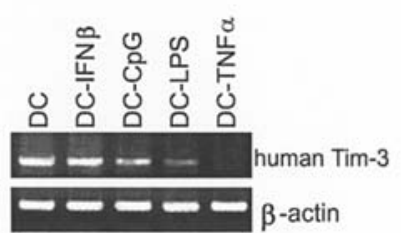

Figure 2. Expression pattern of human Tim-3 in human tissues and cells. (A) Northern blot analysis for tissue distribution of human Tim-3 mRNA. The blot was probed with a probe corresponding to human Tim-3 ORF. (B) RTPCR analysis of human Tim-3 expression with human Tim-3- and B-actinspecific primers on human cells and cell lines. (C) Inducible expression pattern of human Tim-3 in DC stimulated with IFNß, CpG, LPS and TNF $\alpha$.

database revealed that it significantly identical to a Tim (T-cell immunoglobulin- and mucin-domain-containing molecule) family member, human Tim-3 with no descriptions of expression pattern and functions (13). It also resembles other Tim proteins, such as human hepatitis A virus cellular receptor-1 (HuHAVcr-1/Tim-1, Fig. 1), mouse Tim-1, mouse HAVcr-2/Tim-3 (13), and rat kidney injury molecule (Kim-1). To a lesser extent, it is also related to human coxsackievirusadenovirus receptor (data not shown).

This protein has the features of typical type I transmembrane protein. It is composed of an N-terminal 22residue signal peptide, an extracellular domain of 182 residues, a 21-residue transmembrane domain, and a 68residue cytoplasmic region. The extracellular domain consists of an $\mathrm{N}$-terminal cysteine-rich (Cys-rich) region (102 residues) followed by a threonine-, serine-, and proline-rich (TSP-rich) region (73 residues) that contains 37\% serine and threonine residues. The Cys-rich region displays great homology to members of the immunoglobulin (Ig) superfamily (IgSF), and the TSP-rich region has the characteristics of mucin-like glycoproteins, just like those in HuHAVcr-1 and HAVcr-1. There are $2 \mathrm{~N}$-glycosylation sites
A

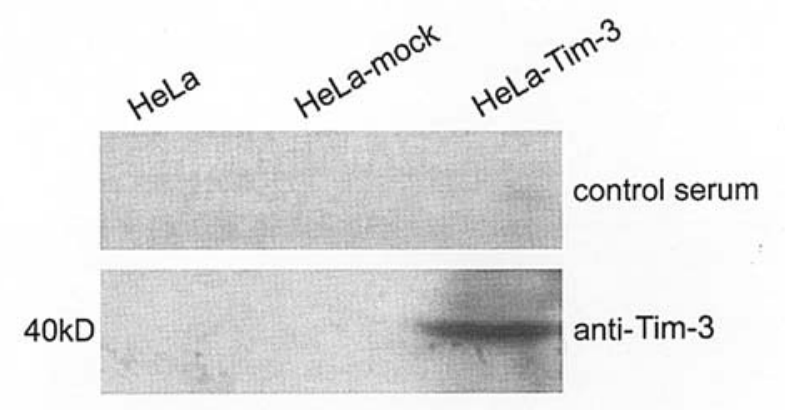

B

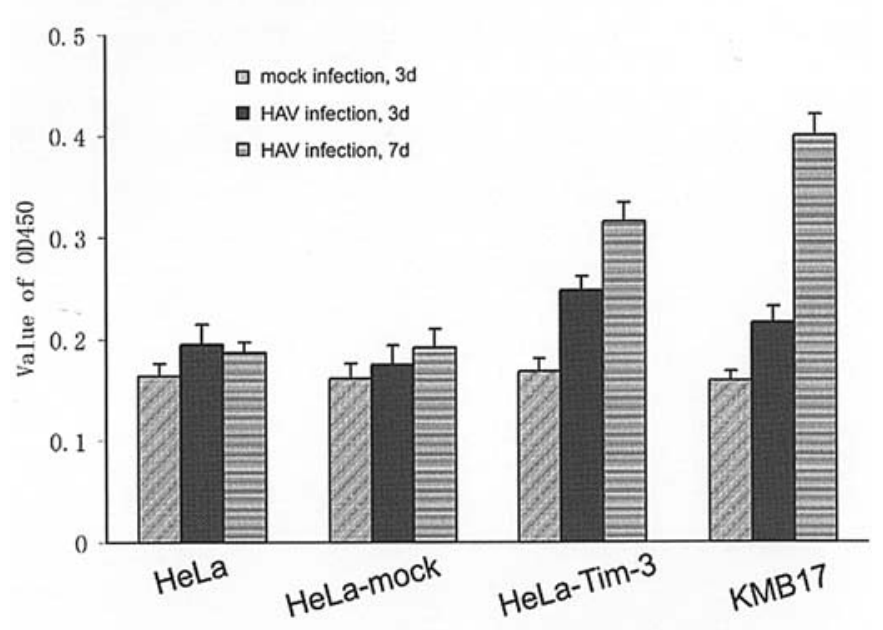

Figure 3. Human Tim-3 promotes HAV entry into target cells. (A) Expression of human Tim-3 on HeLa cells. HeLa cells stably transfected with human Tim-3 expression vector (HeLa-Tim-3) or mock vector (HeLamock) were lyzed and human Tim-3 expression was confirmed by Western blot analysis with anti-Tim-3 polyclonal antibody. (B) Human Tim-3 promotes HAV entry into HeLa cells that overexpress human Tim-3. Cells were infected with HAV H2 strain or mock infectant. After 3 or 7 days of culture, HAV released from the infected cells was tested by ELISA. ${ }^{*} \mathrm{P}<0.05$ vs. HeLa-mock control.

in the extracellular domain. The mucin domain has been proposed to function as a structural domain to present/expose adjacent domains, i.e. it could function to expose the Ig domain well above the plasma membrane as suggested by the 'lollipop on a stick' model (19). As in HuHAVcr-1, six conserved Cys residues were found in its Cys-rich $\mathrm{NH} 2$-terminal Ig-like domain, which are thought to be required for the binding of HAV of HuHAVcr-1 (Fig. 1). One conserved Cys residue (Cys210) was also found within its transmembrane domain, which is possibly used for the addition of fatty acids for stabilizing the receptor attachment to membrane. The cytoplasmic region contained six tyrosines, one of which is part of a tyrosine phosphorylation motif (RSEENIY). The sequence of human Tim-3 was deposited in GenBank with acc. no.: AF251707. 
A

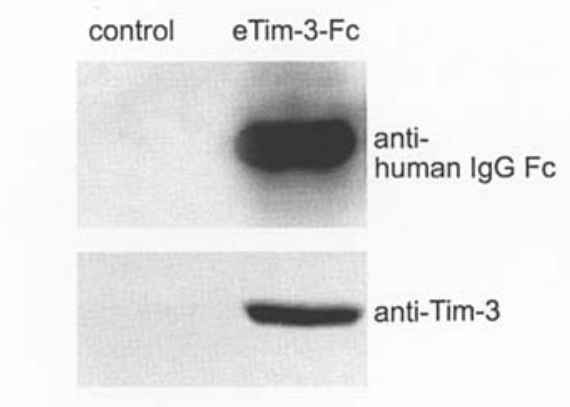

B

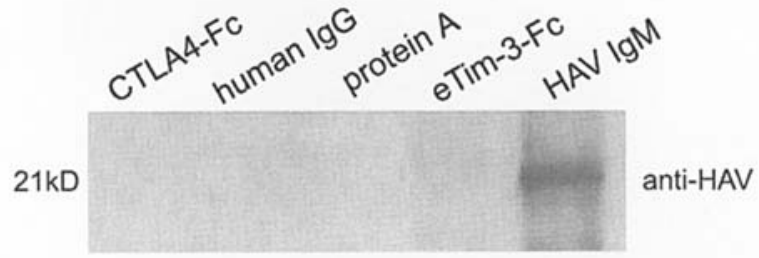

Figure 4. eTim-3-Fc fusion protein cannot bind with HAV directly. (A) Western blot analysis of the purified eTim-3-Fc fusion protein with antihuman IgG Fc antibody and anti-Tim-3 polyclonal antibody. (B) eTim-3-Fc fusion protein cannot bind with HAV directly. Purified eTim-3-Fc protein bound to protein A-Trisacryl beads was incubated with purified HAV. Beads bound HAV were identified by Western blot analysis using anti-HAV antibody.

Analysis for human Tim-3 mRNA expression. The mRNA expression of human Tim-3 in normal human tissues was determined by Northern blot analysis (Fig. 2A). Three transcripts of human Tim-3 were present on Clontech MTN blots. The most ubiquitously expressed was the $2.2-\mathrm{kb}$ transcript, present in all the tissues detected except small intestine, including brain, heart, skeletal muscle, colon, thymus, spleen, kidney, liver, placenta, lung and peripheral blood leukocyte, with the highest expression level in kidney. This $2.2-\mathrm{kb}$ transcript is coherent with the length of human Tim-3 cDNA sequence. A more weaker $7.0-\mathrm{kb}$ transcript was expressed in colon and thymus and a $1.0-\mathrm{kb}$ transcript was seen only in placenta. This mRNA expression pattern of human Tim-3 in normal human tissues was very similar to that of HuHAVcr-1, in which huHAVcr-1 was expressed in nearly every organ analyzed and expressed at higher levels in the kidney than in other organs.

We also examined its cellular distribution by RT-PCR analysis in various hematopoietic cells or cell lines. As shown in Fig. 2B, human Tim-3 mRNA was expressed in THP-1 (myelomonocytic leukemia), U937 (monocytic leukemia), freshly isolated peripheral monocytes and PBMC-derived DC. The results indicated that human Tim-3 might be relatively highly expressed on monocytes or monocyte-derived cell lines. No expression was detected in any of the T cells or B cells (Fig. 2B), which is consistent with the result that mouse Tim-3 is not or lowly expressed in naïve T or B cells (13).

We further examined inducible expression of human Tim-3 mRNA expression in human monocyte-derived DC after different stimulations. It is well known that DC can be matured and activated upon ligation of TNF- $\alpha \mathrm{R}$, or Toll-like receptors. Upon stimulation by $\mathrm{LPS}, \mathrm{CpG}$, and $\mathrm{TNF} \alpha$, human Tim-3 expression was down-regulated significantly, but IFN- $\beta$ stimulation of DC shows no significant influence on expression of human Tim-3 (Fig. 2C). Taken together, the results suggested that antigen-presenting cells such as DC expressed much less human Tim-3 undergoing maturation and activation.

HeLa cells acquire infectivity to HAV after transfection of human Tim-3. It has been shown that HeLa cells, a human Tim-3 mRNA negative cell line, are refractory to HAV infection, but acquire limited level of susceptibility to HAV after transfection of simian HAV receptor. Therefore, HeLa cells were used to analyze the HAV receptor function of human Tim-3. Mammalian expression vector of human Tim-3 was transfected into HeLa cells and a $\sim 40 \mathrm{kDa}$ protein was detected in the HeLa-Tim-3 transfectant, but not HeLa-mock transfectant by Western blot analysis using a polyclonal antibody against human Tim-3 (Fig. 3A). We next infected monolayers of HeLa-Tim-3, HeLa-mock, parental HeLa and KMB 17 cells (as positive control) with HAV or mock infectant. After 3 or 7 days of culture, HAV was released by 3 freeze-thaw cycles from infected cells and the released virus was tested by ELISA. As shown in Fig. 3B, at day 3, absorbance of HeLa-Tim-3 infected by HAV was much greater than that of HAV-infected HeLa-mock and parental HeLa cells, or mock infected cells $(\mathrm{P}<0.05)$. At day 7 , the released virus in HAV- infected HeLa-Tim-3 cells was nearly 2 times more than that of HAV-infected HeLa-mock and HeLa cells $(\mathrm{P}<0.05)$. On the other hand, at day 3, the absorbance of HeLa-Tim-3 and KMB 17 have no significant difference; but at day 7, the absorbance of KMB 17 increased remarkably, and that of HeLa-Tim-3 increased only moderately, suggesting that the overexpression of human Tim-3 in HeLa cells makes the host cells susceptible to HAV infection, but it is very limited. It is possible that human Tim-3 functions as a membrane molecule helping HAV in binding and entering the HeLa cells.

eTim-3-Fc fusion protein cannot bind HAV directly. We analyzed whether human Tim-3 would be a novel cellular receptor of HAV. The extracellular region of human Tim-3 was fused to $\mathrm{Fc}$ region of human $\operatorname{IgG}(\mathrm{eTim}-3-\mathrm{Fc})$ and was expressed on COS7 cells. The fusion protein was purified by affinity chromtography using protein A-agarose column and was confirmed by Western blot analysis. A major 70-kD band, most probably representing a glycosylated form (Fig. 4A) was detected by anti-Fc and anti-Tim-3 probing.

For HAV-binding assay, purified eTim-3-Fc bound to protein A-Trisacryl beads was incubated with purified HAV. Beads bound to HAV were identified by Western blot analysis using anti-HAV antibody. Purified extracellular region of CTLA4 fused with IgG Fc region (CTLA4-Fc) and human $\operatorname{IgG}$ were used as negative controls, and HAV antigen- 
specific IgM was used as a positive control. As shown in Fig. 4B, no HAV was detected on eTim-3-Fc, CTLA4-Fc and the normal human IgG bound beads. However, strong band of HAV was detected bound to human anti-HAV IgM. Together with the fact that the overexpression of human Tim-3 makes the host cells susceptible to HAV infection, the results demonstrated that human Tim-3 does not have direct activity on HAV-binding, only helps HAV to enter the target cells.

\section{Discussion}

DCs constitute the major population of antigen-presenting cells and possess potent ability to initiate $\mathrm{T}$ cell immunity (20). The surface receptors play an important role in DC functions, especially in capturing antigens, contacting with $\mathrm{T}$ cells $(21,22)$. DCs play a central role during virus infections. They may distribute virus from peripheral entry sites towards lymphatic tissue; and infection of DC may pivotally result either in immunosuppression or in immune activation, depending on the cytopathic effect (CPE) of the virus. DCs are among the first cells encountered by viruses, and can be infected by viruses such as LCMV (23), Lassa fever virus $(24,25)$, human cytomegalovirus (26-28), Influenza virus (29), and Measles virus $(30,31)$. Many of them can finish complete life cycles in DCs and also produce an infectious virus. Receptors of viruses on DCs have been identified; $\alpha$-dystroglycan $(\alpha-D G)$ was indentified as the receptor for LCMV (32), and among the immune cells, $\alpha-\mathrm{DG}$ was primarily expressed on CD $11 \mathrm{c}^{+}$and DEC-205+ splenic DC. Some surface molecules of DCs also can act as a viral receptor or coreceptor and are related with viral attachment and infection. DC-SIGN, a type-II membrane protein with a C-terminal C-type lectin domain, has been shown to be a specific and efficient HIV attachment factor (33). The Ig superfamily member ICAM-1 (CD54), which was one of ligands for LFA-1 (CD11a), is expressed on DCs and is identified as a receptor of rhinovirus (34). In this study, we identified human Tim-3, a Ig-like domain-containing IgSF member, from human DC, which shows significant homology with HuHAVcr-1/Tim-1 $(35,36)$, mouse HAVcr-2/Tim-3, and rat Kim-1/Tim-1, all of which are cell surface glycoproteins with common structural motifs, including a signal peptide, Ig domain, mucin domain, transmembrane region and intracellular tail with phosphorylation sites. HuHAVcr-1, the human homologue of HAVcr-1, is a binding receptor as well as a functional receptor for HAV (9). Therefore, we wondered if any relationship exists between HAV infection and this DC surface membrane receptor.

Function of human Tim-3 was analyzed by an infectivity assay. We infected monolayers of human Tim-3overexpressing HeLa cells (HeLa-Tim-3) with HAV. After 3 or 7 days of culture, HAV released by host cells were tested. We showed that HAV inoculation of HeLa cell transfectants expressing human Tim-3 resulted in increased release of $\mathrm{HAV}$, which lasted at least 7 days, indicating that the HeLa cell transfectants gained a limited level of susceptibility to HAV infection. Thus, the human Tim-3 might function as a membrane molecule that can help HAV enter the HeLa cells. We analyzed whether human Tim-3 would be a novel cellular receptor of $\mathrm{HAV}$, mediating the HAV-binding directly. The
eTim-3-Fc fusion protein was prepared for binding with HAV. Our results showed that HAV did not bind directly to eTim-3-Fc, nor did the negative control (CTLA4-Fc and the normal human immunoglobulins). However, HAV bound strongly to the human anti-HAV IgM, which showed that the human anti-HAV Ab specifically detected the HAV antigen, suggesting that eTim-3-Fc did not have direct HAV-binding activity. These lines of evidence strongly suggested that, even if it can promote HAV entry into target cells, human Tim-3 itself cannot function as a cellular receptor of HAV. It has been shown that the Cys-rich Ig-like region of HuHAVcr-1 and its first $\mathrm{N}$-glycosylation site are required for the binding of HAV and certain protective MAb (37). For Tim-3, although it contained the Cys-rich Ig-like domain, the conserved $\mathrm{N}$ glycosylation site was absent, which might partly explain the lack of receptor function of human Tim-3.

The family of T-cell immunoglobulin domain and mucin domain (Tim) proteins was identified with common structural motifs (12). HuHAVcr-1/Tim-1 was considered as a membrane protein that is associated with the development of Th2 biased immune responses and may be selectively expressed on Th2 cell $(12,38)$. Kumanogoh et al reported that mouse Tim-2 interacts with the class IV semaphorin Sema4A, which enhances the activation and differentiation of T cells (39). A study on mouse HAVcr-2/Tim-3 demonstrated that it could regulate macrophage activation and/or function and may have an important role in the induction of autoimmune diseases (13). Others provided the hypothesis that the interaction of mouse Tim-3 and its ligand may be an inhibitory one, serving to limit the expansion of Th1 cell populations and contribute to induction of tolerance of Th1 cells (40). There is no previous study on the biological functions of human Tim-3, especially on the association with viral infection. We demonstrated that, except for Tim-1/HuHAVcr-1, human Tim-3 is related to HAV infection. Given that mouse HAVcr-2/Tim-3 was identified as a negative regulator of Th1 immune response, we can speculate that human Tim-3 might also transduct an inhibitory signal to the cells expressing them (e.g. DC and monocytes), facilitating HAV entry and resulting in suppression of DC and monocyte functions.

\section{Acknowledgements}

We sincerely thank Mrs. Mei Jin, Yan Li, Xianwei Ma and Xiaoting Zuo for their excellent technical assistance. This study was supported by grants from the National Natural Science Foundation of China (No. 30121002), National Key Basic Research Program of China (No. 2001CB510002) and National High Biotechnology Program of China (No. 2002BA711A01).

\section{References}

1. Jennifer C: Hepatitis A: old and new. Clin Microbiol Rev 14: 38-58, 2001.

2. Lemon SM, Jansen RW and Brown EA: Genetic, antigenic and biological differences between strains of hepatitis A virus. Vaccine 10: S40-S44, 1992.

3. Robertson BH, Brown VK and Khanna B: Altered hepatitis A VP1 protein resulting from cell culture propagation of virus. Virus Res 13: 207-212, 1989.

4. Cohen JI, Rosenblum B, Ticehurst JR, Daemer RJ, Feinstone SM and Purcell RH: Complete nucleotide sequence of an attenuated hepatitis A virus: comparison with wild-type virus. Proc Natl Acad Sci USA 84: 2497-2501, 1987 
5. Cohen JI, Rosenblum B, Feinstone SM, Ticehurst J and Purcell RH: Attenuation and cell culture adaptation of hepatitis A virus HAV: a genetic analysis with HAV cDNA. J Virol 63 : 5364-5370, 1989

6. Dotzauer A, Feinstone SM and Kaplan G: Susceptibility of nonprimate cell lines to hepatitis A virus infection. J Virol 68: 6064-6068, 1994

7. Frings $\mathrm{W}$ and Dotzauer A: Adaptation of primate cell-adapted hepatitis A virus strain HM175 to growth in guinea pig cells is independent of mutations in the 5 ' nontranslated region. J Gen Virol 82: 597-602, 2001

8. Kaplan GG, Totsuka P, Thompson T, Akatsuka Y, Moritsugu S and Feinstone M: Identification of a surface glycoprotein on African green monkey kidney cells as a receptor for hepatitis A virus. EMBO J 15: 4282-4296, 1996.

9. Feigelstock D, Thompson P, Mattoo P and Kaplan GG: Polymorphisms of the hepatitis A virus cellular receptor 1 in African green monkey kidney cells result in antigenic variants that do not react with protective monoclonal antibody 190/4. J Virol 72: 6218-6222, 1998.

10. Feigelstock D, Thompson P, Mattoo P, Zhang Y and Kaplan GG: The human homolog of HAVcr-1 codes for a hepatitis A virus cellular receptor. J Virol 72: 6621-6628, 1998.

11. Silberstein E, Dveksler G and Kaplan GG: Neutralization of hepatitis A virus HAV by an immunoadhesin containing the cysteine-rich region of HAV cellular receptor-1. J Virol 75: 717-725, 2001

12. Mclntire JJ, Umetsu SE, Akbari O, et al: Identification of Tapr an airway hyperreactivity regulatory locus and the linked Tim gene family. Nat Immunol 2: 1109-1116, 2001

13. Monney L, Sabatos CA, Gaglia JL, et al: Th1-specific cell surface protein Tim-3 regulates macrophage activation and severity of an autoimmune disease. Nature 415: 536-541, 2002

14. Cao X, Zhang W, Wan T, et al: Molecular cloning and charcterization of a novel CXC chemokine macrophage inflammatory Protein- $2 \gamma$ chemoattractant for human neutrophils and dendritic cells. J Immunol 165: 2588-2595, 2000

15. Zhang W, Wan T, Li N, et al: Genetic approach to insight into the immunobiology of human dendritic cells and identification of CD84-H1, a novel CD84 homologue. Clin Cancer Res 7: S822-S829, 2001.

16. Li N, Zhang W, Wan T, et al: Cloning and characterization of siglec-10, a novel sialic acid-binding member of the Ig superfamily, from human dendritic cells. J Biol Chem 276: 28106-28112, 2001

17. Mao JS: Development of live, attenuated hepatitis A vaccine H2-strain. Vaccine 8: 523-524, 1990

18. Mao JS, Dong DX, Zhang HY, et al: Primary study of attenuated live hepatitis A vaccine H2 strain in humans. J Infect Dis 159: 621-624, 1989.

19. Jentoft N: Why are proteins O-glycosylated? Trends Biochem Sci 15: 291-294, 1990

20. Banchereau J and Steinman RM: Dendritic cells and the control of immunity. Nature 392: 245-252, 1998

21. Sallusto F and Lanzavecchia A: Mobilizing dendritic cells for tolerance, priming, and chronic inflammation. J Exp Med 189: 611-616, 1999 .

22. Steinman RM, Pack M and Inaba K: Dendritic cells in the T-cell areas of lymphoid organs. Immunol Rev 156: 25-32, 1997.

23. Sevilla N, Kunz S, Holz A, et al: Immunosuppression and resultant viral persistence by specific viral targeting of dendritic cells. J Exp Med 192: 1249-1260, 2000.
24. Baize S, Kaplon J, Faure C, Pannetier D, Georges-Courbot MC and Deubel V: Lassa virus infection of human dendritic cells and macrophages is productive but fails to activate cells. J Immunol 172: 2861-2869, 2004.

25. Moutaftsi M, Mehl AM, Borysiewicz LK and Tabi Z: Human cytomegalovirus inhibits maturation and impairs function of monocyte-derived dendritic cells. Blood 99: 2913-2921, 2002.

26. Hertel L, Lacaille VG, Strobl H, Mellins ED and Mocarski ES Susceptibility of immature and mature Langerhans cell-type dendritic cells to infection and immunomodulation by human cytomegalovirus. J Virol 77: 7563-7574, 2003.

27. Riegler S, Hebart H, Einsele H, Brossart P, Jahn G and Sinzger C: Monocyte-derived dendritic cells are permissive to the complete replicative cycle of human cytomegalovirus. J Gen Virol 81: 393-399, 2000

28. Grigoleit U, Riegler S, Einsele H, et al: Human cytomegalovirus induces a direct inhibitory effect on antigen presentation by monocyte-derived immature dendritic cells. Br J Haematol 119: 189-198, 2002.

29. Brimnes MK, Bonifaz L, Steinman RM and Moran TM: Influenza virus-induced dendritic cell maturation is associated with the induction of strong $\mathrm{T}$ cell immunity to a coadministered, normally nonimmunogenic protein. J Exp Med 198: 133-144, 2003.

30. Grosjean I, Caux C, Bella C, et al: Measles virus infects human dendritic cells and blocks their allostimulatory properties for CD4+ T cells. J Exp Med 186: 801-812, 1997.

31. Schnorr JJ, Xanthakos S, Keikavoussi P, et al: Induction of maturation of human blood dendritic cell precursors by measles virus is associated with immunosuppression. Proc Natl Acad Sci USA 94: 5326-5331, 1997.

32. Cao W, Henry MD, Borrow P, et al: Identification of alphadystroglycan as a receptor for lymphocytic choriomeningitis virus and Lassa fever virus. Science 282: 2079-2081, 1998.

33. Geijtenbeek TB, Kwon DS, Torensma R, et al: DC-SIGN, a dendritic cell-specific HIV-1-binding protein that enhances trans-infection of T cells. Cell 100: 587-597, 2000.

34. Olson NH, Kolatkar PR, Oliveira MA, et al: Structure of a human rhinovirus complexed with its receptor molecule. Proc Natl Acad Sci USA 90: 507-511, 1993.

35. Ichimura $\mathrm{T}$, Bonventre JV, Bailly V, et al: Kidney injury molecule-1 KIM-1, a putative epithelial cell adhesion molecule containing a novel immunoglobulin domain, is up-regulated in renal cells after injury. J Biol Chem 273: 4135-4142, 1998.

36. Ichimura T, Hung CC, Yang SA, Stevens JL and Bonventre JV: Kidney injury molecule-1: a tissue and urinary biomarker for nephrotoxicant-induced renal injury. Am J Physiol Renal Physiol 286: F552-F563, 2004

37. Thompson P, Lu J and Kaplan GG: The Cys-rich region of the hepatitis A virus cellular receptor $1 \mathrm{HAVcr}-1$ is required for binding of hepatitis A virus and protective monoclonal antibody 190/4. J Virol 72: 3751-3761, 1998

38. Umetsu DT, Mclntire JJ, Akbari O, Macaubas C and De Kruyff RH: Asthma: an epidemic of dysregulated immunity. Nat Immunol 3: 715-720, 2002.

39. Kumanogoh A, Marukawa S, Suzuki K, et al: Class IV semaphorin Sema4A enhances T-cell activation and interacts with Tim-2. Nature 419: 629-633, 2002.

40. Sabatos CA, Chakravarti S, Cha E, et al: Interaction of Tim-3 and Tim-3 ligand regulates $\mathrm{T}$ helper type 1 responses and induction of peripheral tolerance. Nat Immunol 4: 1102-1110, 2003. 